\title{
Combined use of fluorescence with a magnetic tracer and dilution effect upon sentinel node localization in a murine model
}

This article was published in the following Dove Press journal:

International Journal of Nanomedicine

\author{
Akihiro Kuwahatal,* \\ Muneer Ahmed ${ }^{2, *}$ \\ Kohei Saeki ${ }^{3}$ \\ Shinichi Chikaki' \\ Miki Kaneko' \\ Wenqi Qiu' \\ Zonghao Xin' \\ Shinji Yamaguchi ${ }^{3}$ \\ Akiko Kaneko' \\ Michael Douek ${ }^{2}$ \\ Moriaki Kusakabe 3,4 \\ Masaki Sekino'
}

'Department of Electrical Engineering and Information Systems, Graduate School of Engineering, The University of Tokyo, Hongo, Bunkyo-ku, Tokyo, Japan; ${ }^{2}$ Division of Cancer Studies, King's College London, Guy's Hospital Campus, Great Maze Pond, London, UK; ${ }^{3}$ Graduate School of Agriculture and Life Sciences, The University of Tokyo, Hongo, Bunkyo-ku, Tokyo, Japan; ${ }^{4}$ Matrix Cell Research Institute Inc., Ibaraki, Japan

*These authors contributed equally to this work

Correspondence: Masaki Sekino Department of Electrical Engineering and Information Systems, Graduate School of Engineering, The University of Tokyo, 7-3-I Hongo, Bunkyo-ku, Tokyo I I3-8656, Japan

$\mathrm{Tel}+8 \mathrm{I} 3584 \mid 7490$

Fax +8I 3 584I 6688

Email sekino@bee.t.u-tokyo.ac.jp
Background: Sentinel node biopsy using radioisotope and blue dye remains a gold standard for axillary staging in breast cancer patients with low axillary burden. However, limitations in the use of radioisotopes have resulted in emergence of novel techniques. This is the first in vivo study to assess the feasibility of combining the two most common novel techniques of using a magnetic tracer and indocyanine green (ICG) fluorescence.

Materials and methods: A total of 48 mice were divided into eight groups. Groups 1 and 2, the co-localization groups, received an injection of magnetic tracers $\left(\right.$ Resovist ${ }^{\circledR}$ and Sienna $+{ }^{\circledR}$, respectively) and ICG fluorescence; distilled water was used as the solvent of ICG. Groups 3 and 4, the diluted injection groups, received an injection of magnetic tracers (Resovist and Sienna+, respectively) and saline for dilution. Groups 5, 6, and 7, the control groups, received magnetic tracer (Resovist, Sienna+) and ICG alone, respectively. Fluorescent intensity assessment and iron quantification of excised popliteal lymph nodes were performed. Group 1', a co-localization group, received an injection of magnetic tracers (Resovist) and $\mathrm{ICG}^{\prime}$ fluorescence: saline was used as the solvent for ICG.

Results: Lymphatic uptake of all tracers was confined to the popliteal nodes only, with co-localization confirmed in all cases and no significant difference in fluorescent intensity or iron content of ex vivo nodes between the groups (except for Group 1'). There was no impact of dilution on the iron content in the diluted Sienna+ group, but it significantly enhanced Resovist uptake $(P=0.005)$. In addition, there was a significant difference in iron content $(P=0.003)$ in Group 1'.

Conclusion: The combination of a magnetic tracer (Resovist or Sienna+) and ICG fluorescence is feasible for sentinel node biopsy and will potentially allow for precise transcutaneous node identification, in addition to accurate intraoperative assessment. This radioisotope-free "combined technique" warrants further assessment within a clinical trial.

Keywords: sentinel node biopsy, combined technique, fluorescence dye, magnetic nanoparticles, dilutional effect

\section{Introduction}

Sentinel node biopsy (SNB) emerged in the early 1990s as a targeted alternative to axillary node clearance for staging the axilla, whereby subcutaneous injection of blue dye and subsequently radioisotopes was used to identify the first draining lymph nodes from the primary malignancy. The absence of metastatic spread to the lymph nodes on SNB allowed for the avoidance of axillary node clearance. The reduced morbidity of the procedure resulted in SNB using a radioactive tracer and blue dye (dual technique) becoming the standard of care for axillary staging in clinically node-negative breast 
cancer patients. The use of the "dual technique" provides a high sentinel node identification rate and low false-negative rate. ${ }^{1}$ However, it is accepted that access to radioisotopes is limited and their associated legislation for handling and disposal restricts use of the "dual technique". ${ }^{2}$ The use of blue dye is not without risk when considering the possibility of anaphylaxis. As a consequence of the drawbacks of the "dual technique", other radioisotope-independent techniques have emerged. ${ }^{3,4}$ The most clinically evaluated within the literature are the magnetic technique - using a magnetic tracer and handheld magnetometer - and indocyanine green immunofluorescence using a photodynamic eye (PDE) camera. ${ }^{4}$ These techniques have been evaluated within meta-analyses and demonstrated to show non-inferiority to the "dual technique". The optimization of magnetic tracers within preclinical settings has also been evaluated in terms of volumes and particle sizes. ${ }^{5-7}$ However, all these analyses have possessed methodological limitations, most strikingly that none has consisted of a robust randomized controlled trial against the gold standard. These novel alternative techniques possess both pros and cons compared to the standard "dual technique" and over each other. It is, therefore, conceivable that as with the combined use of radioisotope and blue dye, multimodal, radioisotope-free techniques could be used to overcome the limitations of any single technique. In order for this to be possible, it is essential that techniques consistently allow for the co-localization of sentinel nodes. Within the protocols of conducted clinical trials, ${ }^{8-10}$ the magnetic tracer (Sienna $+{ }^{\circledR}$; Endomagnetics, Cambridge, UK) has been diluted in normal saline for injection. This has increased the volume administered and evidence suggests it may impact adversely upon residual iron remnants on magnetic resonance imaging. ${ }^{11}$ The ability of indocyanine green (ICG) fluorescence and magnetic tracers to co-localize sentinel nodes and the impact of dilution of magnetic tracers were evaluated within a murine model in this study.

\section{Materials and methods}

\section{Tracers}

Three tracers were used in this study: 1) the superparamagnetic iron oxide tracer, Resovist ${ }^{\circledR}$ (Bayer Pharma AG, Tokyo, Japan), containing a magnetic core of maghemite $\left(\gamma-\mathrm{Fe}_{2} \mathrm{O}_{3}\right)$ covered with a carboxydextran coating and an iron concentration of $28 \mathrm{mg} / \mathrm{mL}$; 2) the superparamagnetic iron oxide tracer, Sienna+ (Endomagnetics); and 3) the fluorescent dye ICG, containing serum protein capable of absorbing nearinfrared light at the wavelength of $805 \mathrm{~nm}$ and emitting it at $\sim 820 \mathrm{~nm}$. The difference between Sienna+ and Resovist is that the latter contains mannitol, resulting in a difference in osmolality.

\section{Animals and surgery}

Ethical permission for animal experimentation was granted by the University of Tokyo Ethical Review Board (reference number KA16-4), and all procedures followed were in accordance with the institutional guidelines and ensured humane care of animals. The animals used were 48 female SLC/ICR mice (Japan SLC, Inc., Tokyo, Japan) of age 8-9 weeks, which were sacrificed under deep anesthesia and the popliteal and inguinal nodes were excised.

\section{Imaging systems}

A PDE camera (UI-3240CP-NIR-DL; iDS, Obersulm, Germany) equipped with focus lens (LM12HC-SW; Kowa Optical Products Co., Ltd., Nagoya, Japan) and longpass filter (LIX810; ASAHI SPECTRA Co., Ltd., Tokyo, Japan), which cuts off the near-infrared light with a wavelength shorter than $810 \mathrm{~nm}$, was used to observe the fluorescence emitted by ICG. A red light-emitting diode with a low-pass filterpassing only near-infrared light below $780 \mathrm{~nm}$ - was used for the excitation of ICG, ensuring the PDE camera obtained fluorescence intensity without the influence of the excitation light coming from the light-emitting diode.

\section{Experimental protocol}

To assess the co-localization of magnetic tracers and ICG, the animals were divided into eight groups, each containing six mice. Mice were anesthetized using a mixture of $2 \%-3 \%$ isoflurane and $300 \mathrm{~mL} / \mathrm{min}$ air via an automatic delivery system (Isoflurane Vaporizer; SN-487; Shinano Manufacturing, Tokyo, Japan) during massages and observations using the PDE camera. The mice were repeatedly monitored during the procedure to maintain adequate depth of anesthesia.

Groups 1 and 2 constituted the magnetic tracers and ICG co-localization sentinel node groups. An injection of $5 \mu \mathrm{L}$ magnetic tracer and $5 \mu \mathrm{L}$ diluted ICG $(5 \mathrm{mg} / \mathrm{mL})$ was given bilaterally into the rear footpad (Figure 1A), followed by 5 min of massage to promote tracer uptake. Fluorescence was immediately observed using the PDE camera to assess lymphatic uptake of the fluorescent tracer (Figure 1B). All mice were subsequently sacrificed $15 \mathrm{~min}$ after the injection, and inguinal and popliteal lymph nodes were excised for iron quantification using a superconducting quantum interference device (SQUID, MPMS-5S; Quantum Design, Inc., San Diego, CA, USA) and the fluorescence intensity was assessed 


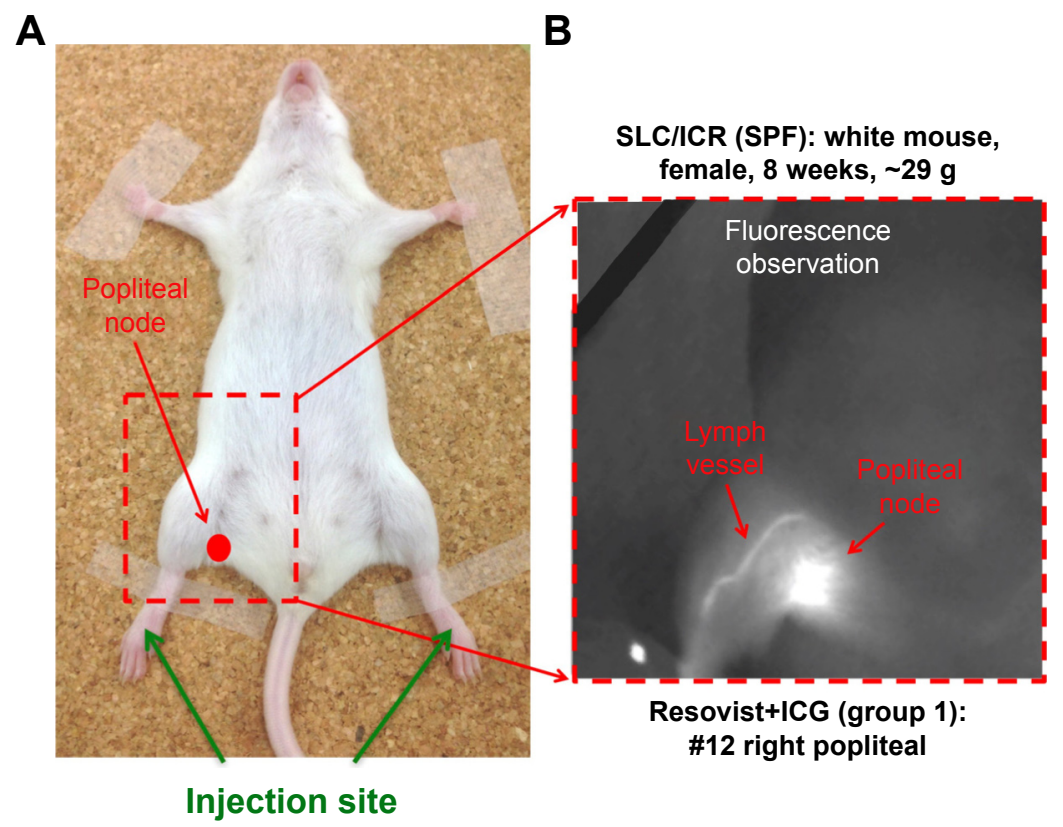

Figure I (A) Location of the injection site and right popliteal node. (B) Transcutaneous fluorescence visible using a near-infrared camera after ICG administration. Abbreviation: ICG, indocyanine green.

using a PDE camera (Figure 2). The magnetic moments of the excised lymph nodes measured by SQUID magnetometry included not only superparamagnetic components of the nanoparticles but also diamagnetic components of the biomedical tissues. To quantify the iron content in the nodes, the magnetic moment of a phantom containing $139 \mu \mathrm{g}$ of iron ( $5 \mu \mathrm{L}$ of Resovist/Sienna+) was used as a reference. Taking into account the subtraction of the diamagnetic components and comparing the measured moments and reference data by use of the least squares method, we obtained the iron content in the nodes. Group 1', in which ICG was diluted in saline instead of distilled water, followed the standard protocol used for groups 1 and 2.

Groups 3 and 4 constituted the diluted magnetic tracer groups. An injection of $5 \mu \mathrm{L}$ of magnetic tracer and $5 \mu \mathrm{L}$ saline ( $100 \%$ dilution, $14 \mathrm{mg} / \mathrm{mL}$ ) was given bilaterally into the rear footpad, followed by $5 \mathrm{~min}$ of massage to promote tracer uptake. The standard protocol applied to groups 1 and 2 was followed, without the assessment of fluorescence.

The other three groups acted as controls. Groups 5 and 6 consisted of mice receiving injection of magnetic tracer $(5 \mu \mathrm{L}$ Resovist and Sienna+, respectively) alone and followed the standard protocol applied to groups 1 and 2, without the assessment of fluorescence. Group 7 consisted of mice injected with $5 \mu \mathrm{L}$ of ICG $(5 \mathrm{mg} / \mathrm{mL})$ alone, and observations using the PDE camera started immediately after the injection and continued for $15 \mathrm{~min}$ to observe the emission intensity of ICG. Inguinal and popliteal lymph nodes were excised and ex vivo quantification analyses of the fluorescence using PDE camera were conducted. Particle size and zeta potential

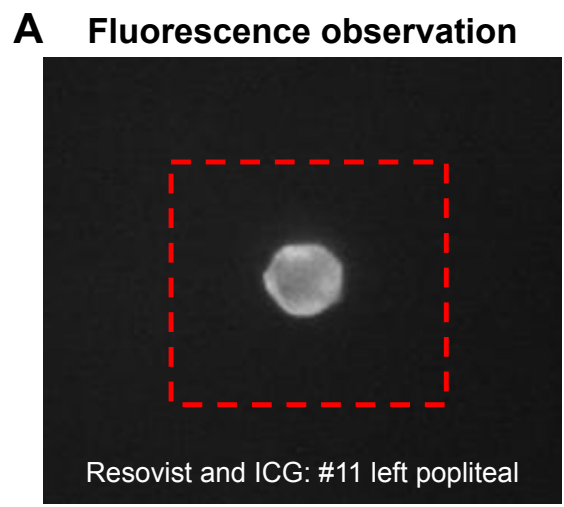

B Visible light observation

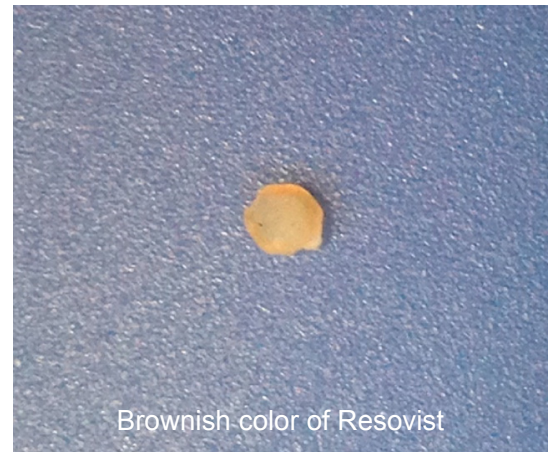

Figure 2 (A) Fluorescence and (B) visible observation of the excised popliteal node in the experiment with Resovist+ICG. Abbreviation: ICG, indocyanine green. 
of each injected tracer were measured by the ELSZ-2 zeta potential and particle size analyzer (Otsuka Electronics Co., Ltd., Osaka, Japan).

\section{Statistical analysis}

Unpaired $t$-tests (SPSS, Chicago, IL, USA) were performed to compare the fluorescent intensity and iron content of ex vivo nodes ( $n=12$ for each group). The relationship between fluorescent intensity and iron content was evaluated using Spearman's correlation.

\section{Results}

\section{Fluorescence intensity}

The magnetic tracer plus ICG (groups 1, 1', and 2) and ICG only (Group 7) groups demonstrated visible fluorescence in bilateral popliteal nodes in all 24 mice. Figure $1 \mathrm{~A}$ and B demonstrates ex vivo node fluorescence and a visible popliteal node - plus lymphatic vessel - transcutaneously on the PDE camera in the combined use of Resovist and ICG, respectively. The mean fluorescence intensities recorded were 29,430 (SD 17,683), 33,354 (SD 21,123), 36,869 (SD 29,843), and 23,987 (SD 12,583) for groups 1, 1', 2 , and 7 , respectively $(P=0.25$ in groups 1 and $7, P=0.11$ in groups $1^{\prime}$ and 7 , and $P=0.13$ in groups 2 and 7 ), as shown in Figure 3A. No uptake was visible in the inguinal lymph nodes, except for one case (unilateral node) using Sienna+ and ICG (Group 2).

\section{Iron quantification}

Groups 1-6 and 1' demonstrated iron uptake by SQUID magnetometry in popliteal nodes only, with a mean iron content $(\mathrm{g})$ of $7.36 \times 10^{-7}\left(\mathrm{SD} 3.25 \times 10^{-7}\right), 13.0 \times 10^{-7}\left(\mathrm{SD} 8.11 \times 10^{-7}\right)$, $20.3 \times 10^{-7}\left(\mathrm{SD} 12.2 \times 10^{-7}\right), 11.3 \times 10^{-7}\left(\mathrm{SD} 10.3 \times 10^{-7}\right)$, $7.44 \times 10^{-7}\left(\mathrm{SD} 5.13 \times 10^{-7}\right), 11.4 \times 10^{-7}\left(\mathrm{SD} 5.78 \times 10^{-7}\right)$, and $14.1 \times 10^{-7}\left(\mathrm{SD} 5.76 \times 10^{-7}\right)$, respectively $(P=0.48$ in groups 1 vs $5, P=0.25$ in groups 2 vs $6, P=0.005$ in groups 3 vs 5 , $P=0.49$ in groups 4 vs $6, P=0.003$ in groups $1^{\prime}$ vs 5 , and $P=0.07$ in groups $1^{\prime}$ vs 1 ), as shown in Figure $3 \mathrm{~B}$ and $\mathrm{C}$. There was no uptake of iron in the inguinal lymph nodes, except for a single node using Sienna+ and ICG (Group 2). Detailed results are shown in Tables 1 and 2 . Iron content in the popliteal nodes was $\sim 1 \%$ of the injection amount $(139 \mu \mathrm{g})$.

\section{Relationship between fluorescence intensity from the node and uptake of magnetic tracers in the node}

Groups 1 and 2 demonstrated a relationship between fluorescence intensity and iron uptake, with the correlation factor $R$ being $\sim 0.40$ (Figure 4 ).
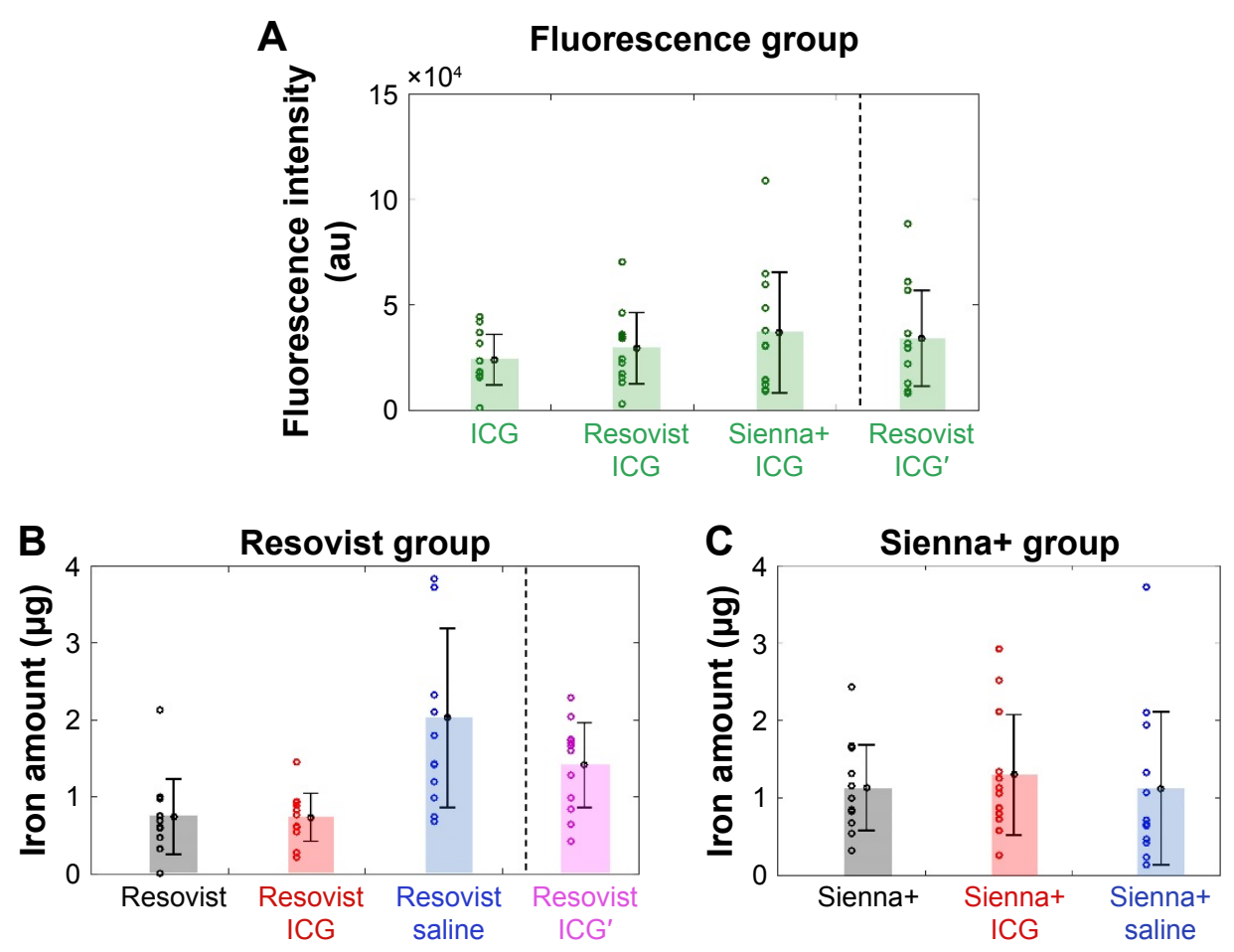

Figure 3 Accumulation of the tracers in the popliteal nodes.

Notes: (A) Fluorescence intensity from the popliteal nodes. (B and C) Iron amount evaluated by SQUID measurements. ICG and ICG' were used with distilled water and saline as the solvent, respectively.

Abbreviations: ICG, indocyanine green; SQUID, superconducting quantum interference device. 
Table I Injection tracer(s), mean iron content, and fluorescent intensity in each group

\begin{tabular}{llll}
\hline Group & Tracer(s) & $\begin{array}{l}\text { Iron } \\
\text { content } \\
\left(\mathbf{I 0}^{-7} \mathbf{~ g )}\right.\end{array}$ & $\begin{array}{l}\text { Fluorescence } \\
\text { intensity (au) }\end{array}$ \\
\hline $\mathrm{I}$ & Resovist $(5 \mu \mathrm{L})$ and ICG $(5 \mu \mathrm{L})$ & $\mathbf{7 . 3 6}$ & $\mathbf{2 9 , 4 3 0}$ \\
2 & Sienna+ $(5 \mu \mathrm{L})$ and ICG $(5 \mu \mathrm{L})$ & $\mathbf{1 3 . 0}$ & $\mathbf{3 6 , 8 6 9}$ \\
3 & Resovist $(5 \mu \mathrm{L})$ and saline $(5 \mu \mathrm{L})$ & 20.3 & - \\
4 & Sienna+ $(5 \mu \mathrm{L})$ and saline $(5 \mu \mathrm{L})$ & 11.3 & - \\
5 & Resovist $(5 \mu \mathrm{L})$ & 7.44 & - \\
6 & Sienna+ $(5 \mu \mathrm{L})$ & $1 \mathrm{II} .4$ & - \\
7 & ICG $(5 \mu \mathrm{L})$ & - & 23,987 \\
$\mathrm{I}^{\prime}$ & Resovist $(5 \mu \mathrm{L})$ and ICG' $(5 \mu \mathrm{L})^{\mathrm{a}}$ & $\mathbf{I 4 . 1}$ & $\mathbf{3 3 , 3 5 4}$ \\
\hline
\end{tabular}

Notes: alCG' was used with saline as the solvent. Bold font represents the colocalization groups.

Abbreviation: ICG, indocyanine green.

\section{Particle size and zeta potential of injected tracers}

Groups 1-6 and 1' demonstrated fairly similar particle size and zeta potential of the injected tracer. Detailed results are shown in Table 3.

\section{Discussion}

SNB is the gold standard for axillary staging in breast cancer patients with suspected low axillary burden. The combined use of radioisotope and blue dye has been demonstrated by meta-analysis to yield the highest sensitivity and lowest false-negative rate compared to either technique used alone. ${ }^{1}$ Novel techniques have emerged as alternatives for SNB, which are radioisotope independent and therefore allow easier access and utilization in isotope-replete areas. This study was conducted to assess the feasibility of combining two radioisotope-independent techniques to form a feasible "combined technique" for SNB. The magnetic and fluorescent (ICG) techniques have been the most extensively evaluated of these novel procedures. A technical limitation

Table $2 P$-values calculated from unpaired $t$-tests

\begin{tabular}{ll}
\hline Groups & $P$-value \\
\hline Resovist, ICG/Resovist & $0.48^{\mathrm{a}}$ \\
Sienna+, ICG/Sienna+ & $0.25^{\mathrm{a}}$ \\
Resovist/Sienna+ & $0.03^{*, \mathrm{a}}$ \\
Diluted Resovist/Resovist & $0.005^{*, a}$ \\
Diluted Sienna+/Sienna+ & $0.49^{\mathrm{a}}$ \\
Resovist, ICG/ICG & $0.25^{\mathrm{b}}$ \\
Sienna+, ICG/ICG & $0.13^{\mathrm{b}}$ \\
Resovist, ICG'/ICG & $0.1 I^{\mathrm{b}}$ \\
Resovist, ICG'/Resovist & $0.003^{* *, a}$ \\
Resovist, ICG'/diluted Resovist & $0.07^{\mathrm{a}}$ \\
\hline
\end{tabular}

Notes: $P$-values of ${ }^{\text {airon }}$ content and ${ }^{b}$ fluorescence intensity. Significant difference, $* P<0.05, * * P<0.005$. ICG' was used with saline as the solvent.

Abbreviation: ICG, indocyanine green.

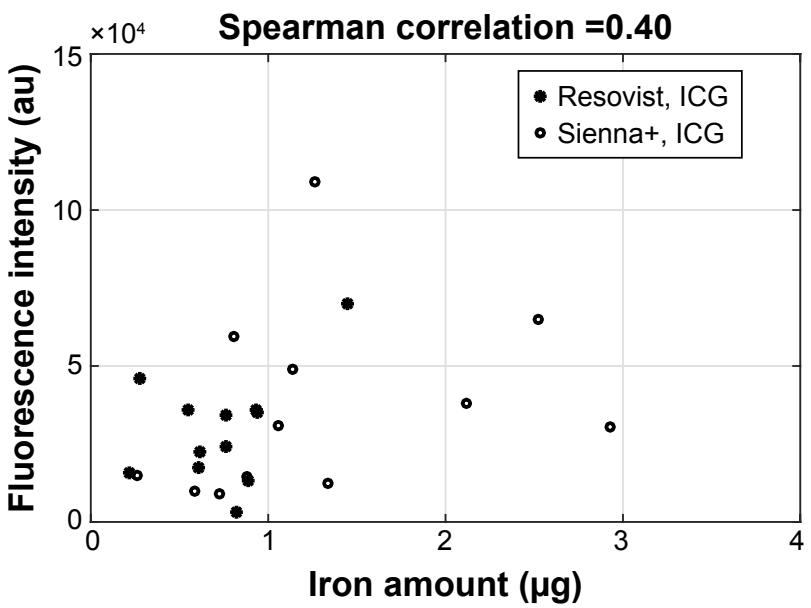

Figure 4 Correlation between uptake of magnetic tracers in the nodes and fluorescence intensity from the node.

Note: Correlation factor $R$ is $\sim 0.40$.

Abbreviation: ICG, indocyanine green.

of the magnetic technique has been the inability to identify a transcutaneous signal (hotspot) to precisely define the incision point preoperatively. This results in the surgeon being more dependent upon signal identification after the skin incision is made. On the contrary, the fluorescent technique allows transcutaneous visualization of sentinel nodes using a PDE camera and precise positioning of a surgical incision. Therefore, by combining these two techniques, it would be considered possible to attain the benefits of transcutaneous fluorescent marking and precise, real-time intraoperative guidance using the handheld magnetometer. However, such an approach is only feasible if there is co-localization of sentinel nodes by the two techniques, otherwise it would result in unnecessary nodal retrieval.

This study has been able to demonstrate that uptake of both tracers was limited to the primary draining lymph node of the murine lower limb (popliteal node) ${ }^{12}$ and did not spread to other locations. This is highly suggestive of an adequate

Table 3 Particle size and zeta potential of the injection tracer(s) in each group

\begin{tabular}{llll}
\hline Group & Tracer(s) & $\begin{array}{l}\text { Particle } \\
\text { size }(\mathbf{n m})\end{array}$ & $\begin{array}{l}\text { Zeta potential } \\
(\mathbf{m V})\end{array}$ \\
\hline $\mathrm{I}$ & Resovist $(5 \mu \mathrm{L})$ and ICG $(5 \mu \mathrm{L})$ & 78.8 & -51.8 \\
2 & Sienna+ $(5 \mu \mathrm{L})$ and ICG $(5 \mu \mathrm{L})$ & 77.9 & -43.5 \\
3 & Resovist $(5 \mu \mathrm{L})$ and saline $(5 \mu \mathrm{L})$ & 78.0 & -44.6 \\
4 & Sienna+ $(5 \mu \mathrm{L})$ and saline $(5 \mu \mathrm{L})$ & 68.5 & -36.0 \\
5 & Resovist $(5 \mu \mathrm{L})$ & 73.5 & -44.9 \\
6 & Sienna+ $(5 \mu \mathrm{L})$ & 72.6 & -39.6 \\
7 & ICG $(5 \mu \mathrm{L})$ & $\mathrm{N} / \mathrm{A}$ & $\mathrm{N} / \mathrm{A}$ \\
$\mathrm{I}^{\prime}$ & Resovist $(5 \mu \mathrm{L})$ and ICG' $(5 \mu \mathrm{L})^{\mathrm{a}}$ & 75.7 & -47.6 \\
\hline
\end{tabular}

Notes: 'a ${ }^{2}$ ' was used with saline as the solvent. These parameters were not measured for ICG because its particle size corresponds to molecular size. Abbreviations: ICG, indocyanine green; N/A, not available. 
dose of tracer being administered to prevent saturation of the primary draining node and passage to higher echelon nodes. In all cases, co-localization of the two tracers occurred without any evidence of drainage to higher echelon nodes, except for one single case. In terms of the fluorescence evaluations, there was no significant difference among groups, indicating that ICG uptakes into the lymph nodes without serious particle interactions. No suggestions of deleterious effects from combining the two tracers were demonstrated, with no significant difference in fluorescence intensity or iron uptake between groups 1, 2, 5, 6, and 7 . This result indicates that the dilutional effect and interaction of the two separate tracers did not result in any adverse effect on nodal uptake. Furthermore, the correlation factor of the relationship between fluorescence intensity and iron uptake is $\sim 0.40$, suggesting the presence of a moderate correlation. In terms of the dilution group, we could not find significant differences in the experiment with Sienna+ $(P=0.49)$. In contrast, a significant difference was found in the diluted Resovist group $(P=0.005)$. This difference between the two magnetic tracers and dilutional effect is likely the result of their differing osmolalities. Resovist possesses an osmolality of $333 \mathrm{mOsm} / \mathrm{kg}$, whereas Sienna+ has a value of $80-160 \mathrm{mOsm} / \mathrm{kg}$. The osmolality of interstitial fluid is in the range of 275-295 $\mathrm{mOsm} / \mathrm{kg}$. The lower osmolality of Sienna+ encourages fluid shifts from the interstitium toward the lymphatics, as was demonstrated by its significantly greater nodal deposition of iron using neat Sienna+ compared to Resovist $(P=0.03)$. This superiority over Resovist may also be attributable to the physiological $\mathrm{pH}$ maintained by the presence of mannitol in its excipients, which influences the osmolality. The osmolality of Sienna+ appears to be the optimal value, as when diluted further, there is no improvement in iron uptake. By contrast, Resovist, with an osmolality of more than twice that of Sienna+, allows for a significant improvement $(P=0.005)$ in nodal iron uptake when diluted, therefore approaching an osmolality closer to that of Sienna+. This suggests that the dilution of Resovist could optimize the tracer for clinical use, as compared to Sienna+, in which case dilution appears unnecessary. In addition, we report significant differences $(P=0.003)$ between Group 1', which used Resovist and $\mathrm{ICG}^{\prime}$ (solvent is saline), and Group 5 using Resovist alone. The uptake of magnetic tracers into the nodes was approximately double the amount with Resovist only. This result shows that the combined use of Resovist and $\mathrm{ICG}^{\prime}$ (solvent is saline) leads to significantly improved detection of sentinel lymph nodes. To investigate the factor that could promote the uptake of magnetic nanoparticles, we measured the particle size and zeta potential of the tracers, but could not identify any particle aggregation and potential changes that would impact the accumulation in the node. Therefore, we conclude that the osmolality change is the most important factor for tracer accumulation in the nodes.

\section{Conclusion}

This is the first in vivo study to assess the use of combining a magnetic and fluorescent tracer for the purposes of SNB. It demonstrates that the technique is feasible, co-localizes sentinel nodes, and avoids higher echelon node involvement. This method represents an alternative radioisotope-free "combined technique" for SNB, which warrants assessment within a clinical trial to determine if it translates to any benefit within the clinical setting.

\section{Acknowledgments}

This work was supported by the Development of Medical Devices and Systems for Advanced Medical Services of the Japan Agency for Medical Research and Development (AMED) and a grant from the Magnetic Health Science Foundation.

\section{Disclosure}

The authors report no conflicts of interest in this work.

\section{References}

1. Kim T, Giuliano AE, Lyman GH. Lymphatic mapping and sentinel lymph node biopsy in early-stage breast carcinoma: a metaanalysis. Cancer. 2006;106(1):4-16.

2. Ahmed M, Douek M. The role of magnetic nanoparticles in the localization and treatment of breast cancer. Biomed Res Int. 2013;2013: 281230.

3. Zada A, Peek MC, Ahmed M, et al. Meta-analysis of sentinel lymph node biopsy in breast cancer using the magnetic technique. Br J Surg. 2016; 103(11):1409-1419.

4. Ahmed M, Purushotham AD, Douek M. Novel techniques for sentinel lymph node biopsy in breast cancer: a systematic review. Lancet Oncol. 2014;15(8):e351-e362.

5. Ahmed M, Anninga B, Pouw JJ, et al. Optimising magnetic sentinel lymph node biopsy in an in vivo porcine model. Nanomedicine. 2015;11(4): 993-1002.

6. Anninga B, Ahmed M, Van Hemelrijck M, et al. Magnetic sentinel lymph node biopsy and localization properties of a magnetic tracer in an in vivo porcine model. Breast Cancer Res Treat. 2013;141(1):33-42.

7. Pouw JJ, Ahmed M, Anninga B, et al. Comparison of three magnetic nanoparticle tracers for sentinel lymph node biopsy in an in vivo porcine model. Int J Nanomedicine. 2015;10:1235-1243.

8. Rubio IT, Diaz-Botero S, Esgueva A, et al. The superparamagnetic iron oxide is equivalent to the Tc99 radiotracer method for identifying the sentinel lymph node in breast cancer. Eur J Surg Oncol. 2015;41(1): $46-51$. 
9. Thill M, Kurylcio A, Welter R, et al. The Central-European SentiMag study: Sentinel lymph node biopsy with superparamagnetic iron oxide (SPIO) vs. radioisotope. Breast. 2014;23(2):175-179.

10. Douek M, Klaase J, Monypenny I, et al; SentiMAG Trialists Group. Sentinel node biopsy using a magnetic tracer versus standard technique: the SentiMAG Multicentre Trial. Ann Surg Oncol. 2014;21(4): 1237-1245.
11. Huizing E, Anninga B, Young P, Monnypenny I, Hall-Craggs M, Douek M. Analysis of void artefacts in post-operative breast MRI due to residual SPIO after magnetic SLNB in SentiMAG Trial participants. Eur J Surg Oncol. 2015;41(6):S18.

12. Ahmed M, Woo T, Ohashi K, et al. Magnetic sentinel lymph node biopsy in a murine tumour model. Nanomedicine. 2016;12(4):1045-1052.
International Journal of Nanomedicine

\section{Publish your work in this journal}

The International Journal of Nanomedicine is an international, peerreviewed journal focusing on the application of nanotechnology in diagnostics, therapeutics, and drug delivery systems throughout the biomedical field. This journal is indexed on PubMed Central, MedLine, CAS, SciSearch $®$, Current Contents $® /$ Clinical Medicine,

\section{Dovepress}

Journal Citation Reports/Science Edition, EMBase, Scopus and the Elsevier Bibliographic databases. The manuscript management system is completely online and includes a very quick and fair peer-review system, which is all easy to use. Visit http://www.dovepress.com/ testimonials.php to read real quotes from published authors.

Submit your manuscript here: http://www.dovepress.com/international-journal-of-nanomedicine-journal 